\title{
Assessment of Nurses' Knowledge about Food Reintroduction in Tracheostomized Patients
}

Maine Raquel Marinho3, Maiza Claudia Vilela Hipólito, Ana Carine Arruda Rolim4, Herla Maria Furtado Jorge ${ }^{5}$, Ana Maria Martins Pereira6, Valeria Aparecida Masson ${ }^{2}$

\section{Abstract}

Objective: Assess the knowledge of nurses working in a hospital regarding food reintroduction to tracheostomized patients.

Method: This is a quantitative, descriptive and cross-sectional study which used an instrument that consists of 20 closed questions, divided into two parts, applied to a sample of 50 clinical nurses in April 2011.

Results: The sample was composed of $90 \%$ of female subjects with mean age of 35.4 years, 37\% of respondents reported having more than ten years of professional experience and $83 \%$ stated that were never approached about the subject and that were unaware of the medical conditions to which the patient should submit to resume feeding by mouth.

Conclusion: It was found professional unpreparedness and lack of knowledge in the subject and that approaching the theme since graduation and continuing education measures to be implemented in institutions that meet tracheostomized patients could provide the reintroduction of oral diet safely.

\section{Keywords}

Nursing Care; Tracheostomy; Feeding Behavior.

\section{Introduction}

The patient in use of tracheostomy requires a functional suction system, oxygen supply, ventilation bag and tracheostomy kit in order to avoid complications such as infection, tracheomalacia, skin breakdown and tracheoesophageal fistula. Some emergency situations may arise, 
which include bleeding, tube displacement, loss of airway and tube obstruction; such emergencies are resolved more effectively when all the necessary supplies are readily available at the bedside [1, 4].

Besides these difficulties, tracheostomy can trigger swallowing disorders, leading to reduced frequency of swallowing and coughing reflexes, reduced laryngeal elevation and anteriorization, decreased pharyngeal and laryngeal sensitivity, atrophy of laryngeal muscles, incoordination and reduced glottal closing time, esophageal compression by the cuff and decreased subglottic pressure. To minimize possible risks, the clinical assessment by a speech therapist is of great value in identifying potential functional changes that interfere with oral and pharyngeal phases of swallowing [3].

In addition to identifying potential functional changes, the clinical assessment by a speech therapist is also needed before the reintroduction of food by mouth to check the patient's swallowing process, so that it is held safely, not to bring harm to the patient, which will regress all the rehabilitation process [5].

The nursing professional is the one who provides and monitors the process of feeding the patient, providing assistance that favors food reintroduction after the clinical assessment by the speech therapist, risk free.

The nurse, responsible for nursing care and as a member of the multidisciplinary team, has the function to provide proper care to prevent complications. They also work in direct care and guidance of health promotion measures. In a hospital, they are near the patient during hospitalization, participating in all stages of care. Therefore, they must also be present throughout the process of reintroducing food [4].

A study conducted in Japan in order to verify the impact of a training program directed to nurses of noncritical areas about the care implemented in tracheostomized patients found decreased rates of complications and readmissions in Intensive Care
Units (ICU) after the implementations of the program [4].

Food reintroduction has always been performed with criteria defined by the multidisciplinary team, often with the aim to meet the sensory needs of the patient requesting water and food, since enteral or parenteral nutrition does not give pleasure as oral feeding. Therefore, food reintroduction without defining criteria and without strict monitoring can cause the patient serious pulmonary risks, such as bronchoaspiration and aspiration pneumonia, causing increased length of stay, costs and even risk of death.

Thus, the present study aims to assess the knowledge of nurses on the process of food reintroduction in tracheostomized patients.

\section{Methodology}

This is a descriptive, quantitative and cross-sectional study $[6,7]$, conducted in a hospital in the state of São Paulo, which has 406 beds and is a reference in the region in the service to users of the Unified Health System (SUS) and in the development of scientific research.

The survey was conducted in the ICU, Postoperative Unit, Coronary Care Unit, Clinical Emergency, Traumatology, Adult General Nursing and Pediatrics, which includes Inpatient Pediatric Unit, Surgical Pediatrics and Pediatric ICU.

Considering the purpose and the design of this study, the population was composed of nurses working in April 2011 in assisting the tracheostomized patients in the described areas and who agreed to participate in this study by signing the Informed Consent Form.

Study participants were 50 nurses who were available and who met the inclusion and exclusion criteria [6]. There was refusal and loss rate of $16 \%$, resulting in a response rate of $82 \%$ of the initial sample. Data collection was carried out by researchers in previously scheduled date, and the subjects 
were instructed to fill out the instrument. They had a period of six hours for day shifts and 12 hours for night shifts to fill it.

The instrument for evaluation of nurses' knowledge on food reintroduction to tracheostomized patients was developed by the authors, therefore, it is entirely original and was composed of 20 closed questions divided into two domains: sociodemographic profile and knowledge on food reintroduction in tracheostomized patients. The results were expressed by descriptive analysis and relative frequency.

The study complied with the ethical requirements in accordance with the provisions of the National Health Council (Resolutions No. 196/96), recommended by the Ethics Committee of the School of Medical Sciences of the State University of Campinas - FCM/UNICAMP and was approved by the Ethics Committee on 11 February 2011, under opinion No. 1258/2010.

\section{Results}

The study included 41 nurses of both sexes, with prevalence of females, composing $90 \%$ of the sample. The age of the subjects ranged from 24 to 55 years old, with a mean of 35.4 years. Of the participating nurses, $51 \%$ had specialization, $26 \%$ improved education, 20\% master's degree and 3\% doctorate. The time of professional practice of the subjects ranged from less than one year and more than ten years, of which $2 \%$ had less than one year, 29\% between one year and five years, 32\% between six and ten years and 37\% with more than ten years of professional practice.

As regards the approach to food reintroduction during the academic education, $83 \%$ of respondents reported not having had contact with this subject throughout their training. Among those who reported contact, $12 \%$ had it during graduation and 5\% during postgraduation. Regarding the training on this subject in the place of work, only $25 \%$ of nurses reported having been trained on this subject.
In relation to the clinical conditions that the patient must present so that food reintroduction is considered, $15 \%$ of subjects pointed the level of consciousness, followed by spontaneous ventilation (10\%), absence of oxygen support (2\%), tolerance to deflated cuff $(7 \%)$, tolerance to decubitus $90^{\circ}$ $(10 \%)$, and others. It is noteworthy that in this question subjects could choose more than one answer.

(Table 1)

Table 1. Distribution of nurses according to the clinical conditions that the patient must present to feed reintroduction (Campinas, 2011).

\begin{tabular}{|l|l|c|c|}
\hline Variables & \multicolumn{1}{|c|}{ Categories } & N & $\%$ \\
\hline & Level of consciousness & 37 & 15 \\
\cline { 2 - 4 } Evaluated & Spontaneous ventilation & 26 & 10 \\
\hline factors & Absence of oxygen support & 6 & 2 \\
\hline & Tolerance to deflated cuff & 17 & 7 \\
\hline & Tolerance to decubitus 90 & 24 & 10 \\
\hline Total & Others & 4 & 2 \\
\hline
\end{tabular}

Regarding the initial evaluation to start reintroduction of oral feeding, when asked which factors must be evaluated, $30 \%$ of subjects pointed the ability to swallow followed by the risk for bronchoaspiration (28\%) and level of consciousness $(27 \%)$ as the factors that must be evaluated before food reintroduction. It is noteworthy that in this question subjects could choose more than one answer. (Table 2)

Table 2. Distribution of nurses according to the conditions that must be monitored in the previous evaluation to start oral feeding in tracheostomized patients (Campinas, 2011).

\begin{tabular}{|l|l|c|c|}
\hline Variables & \multicolumn{1}{|c|}{ Categories } & N & $\%$ \\
\hline & Ability to swallow & 13 & 30 \\
\hline \multirow{3}{*}{ Evaluated } & Risk for bronchoaspiration & 11 & 28 \\
\hline factors & Level of consciousness & 11 & 27 \\
\hline & Satisfaction in eating & 5 & 14 \\
\hline Total & Others & 1 & 1 \\
\hline
\end{tabular}


When asked which professional should perform the preliminary assessment for the start of oral feeding, $42 \%$ of subjects responded that this evaluation should be performed by the speech therapist, $24 \%$ by the multidisciplinary team, $14 \%$ by nurses, $8 \%$ by physicians, $6 \%$ by the nutritionist and $6 \%$ by the physical therapist.

With regard to the professional who must indicate the beginning of food reintroduction by mouth to tracheostomized patients, $47 \%$ of nurses stated that the initial indication should be performed by a multidisciplinary team, followed by the speech therapist (22\%), physician (12\%), nutritionist (10\%), nurse $(9 \%)$ and physical therapist $(0 \%)$. It is noteworthy that in this question subjects could choose more than one answer.

When asked about the frequency of revaluation after starting the diet, $47 \%$ of subjects answered that such evaluation should always take place before the offering of each diet. Subjects were asked about the frequency with which the oral feeding is reintroduced for tracheotomized patients, and most reported that this practice rarely happens (60\%). Regarding the use of protocol for food reintroduction, $100 \%$ of respondents found it useful. This can be proved through the testimonies:

Because eating is a basic human need, reintroducing oral feeding as soon as possible may decrease the time of care provided, and the patient can be healthy again as quickly as possible.

25, Campinas.

In order to have a greater understanding of the subject and to have a protocol that we all can meet.

27, Campinas.

To minimize possible complications during offering diet by mouth.

28, Campinas.
So that all nurses within the institution are trained, and to conduct it safely to both.

32, Campinas.

During the offering of the diet, several factors must be observed. The return of the diet through the tracheostomy and the respiratory frequency $(24 \%, 23 \%)$ were the parameters most chosen by nurses. (Table 3)

Table 3. Distribution of nurses according to the parameters observed during the offering of the diet by mouth (Campinas, 2011).

\begin{tabular}{|c|c|c|c|}
\hline Variables & Categories & $\mathbf{N}$ & $\%$ \\
\hline \multirow{7}{*}{$\begin{array}{l}\text { Observed } \\
\text { parameters }\end{array}$} & Respiratory frequency & 36 & 15 \\
\hline & Heart rate & 12 & 10 \\
\hline & Blood pressure & 4 & 2 \\
\hline & Saturation & 32 & 7 \\
\hline & Return of the diet & 37 & 10 \\
\hline & Patient acceptance & 31 & 2 \\
\hline & Others & 2 & 10 \\
\hline \multicolumn{2}{|l|}{ Total } & 41 & 100 \\
\hline
\end{tabular}

Among these complications, $41 \%$ of subjects mentioned the aspiration pneumonia as one of the complications that could arise. Respondents could choose more than one answer. (Table 4)

Table 4. Distribution of nurses according to the clinical complications of food reintroduction without defined criteria (Campinas, 2011).

\begin{tabular}{|l|l|c|c|}
\hline Variables & \multicolumn{1}{|c|}{ Categories } & N & $\%$ \\
\hline & Aspiration pneumonia & 17 & 41 \\
\hline & $\begin{array}{l}\text { Bronchoaspiration } \\
\text { Silent aspiration }\end{array}$ & 16 & 39 \\
\hline $\begin{array}{l}\text { Evaluated } \\
\text { factors }\end{array}$ & $\begin{array}{l}\text { Acute Respiratory } \\
\text { Insufficiency }\end{array}$ & 6 & 15 \\
\hline & $\begin{array}{l}\text { Systemic Respiratory } \\
\text { Insufficiency }\end{array}$ & 0 & 0 \\
\hline Total & $\begin{array}{l}\text { Sepsis } \\
\text { Others }\end{array}$ & 1 & 3 \\
\hline
\end{tabular}


Table 5. Distribution of nurses according to the parameters to be monitored throughout the process of feeding reintroduction (Campinas, 2011).

\begin{tabular}{|l|l|c|c|}
\hline Variables & \multicolumn{1}{|c|}{ Categories } & N & $\%$ \\
\hline \multirow{3}{*}{ Monitored } & Vital signs & 12 & 30 \\
\hline parameters & Chest X-ray & 12 & 29 \\
\cline { 2 - 4 } & Laboratory tests & 1 & 4 \\
\cline { 2 - 4 } & Hydric balance & 4 & 9 \\
\cline { 2 - 4 } & Ventilatory parameters & 11 & 26 \\
\hline Total & Computed tomography & 1 & 2 \\
\hline
\end{tabular}

To prevent any complications throughout the process of reintroducing food, $30 \%$ of the subjects believe that vital signs followed by chest X-ray (29\%) should be monitored parameters throughout the process. Respondents could choose more than one answer. (Table 05)

\section{Discussion}

Food reintroduction in tracheostomized patients is not a commonly discussed subject during the academic nursing training, which causes that the professional does not have necessary knowledge on the process as a whole [8]. It is known that the use of tracheostomy is common in patients who require intensive care and that the improvement of their status may present the parameters required for food reintroduction, but for this to be realized by the team, it is necessary that all have adequate knowledge, which should begin during graduation.

Another important factor in the process of food reintroduction is that there is a protocol and training of professionals [9]. The university to which the hospital is linked created and implemented the project in the Intensive Care Unit in 2010 from a demand of the multidisciplinary team front the need to standardize the conduct, and due to the lack of speech therapists in the unit teams [10].
There is a consensus in the literature that, to feed reintroduction, the patient must present three concurrent medical conditions, which are level of consciousness (glasgow $>=12$ ), toleration to deflated cuff and decubitus $90^{\circ}$ [11, 12]. Although subjects could choose more than one answer, none of the participants indicated only these three alternatives concurrently.

Studies show that food reintroduction without defined criteria can lead to a worsening of the clinical condition of the patient, which may be evidenced by several complications [11, 12]. Among these complications, $41 \%$ of subjects mentioned aspiration pneumonia as one of the complications that could arise.

The American Speech-Language-Hearing Association (ASHA) reports that the assessment should be carried out in three steps, where each step is performed in a different day. Professionals must evaluate the ability to swallow, observe the risk for bronchoaspiration and patient satisfaction in eating [11]. Nurses have mostly showed this need in the evaluation of swallowing ability and risk for bronchoaspiration, but patient satisfaction in eating was a barely mentioned item, which shows that the implications that denote individuals' autonomy are often forgotten or poorly understood by professionals.

In the whole process of food reintroduction, the patient must be reevaluated before the offering each diet because their clinical conditions may change in a short time due to severe situations, medication and other factors that can quickly change their current framework [9]. Despite the inadequate approach on the subject, the vast majority of subjects mentioned that would reassess patients before offering each diet and pointed the nurse as the most appropriate professional to perform this action.

Corroborating the above statement, literature review of 2014 showed that nurses who care for patients with tracheostomy have breadth of knowledge required to provide individual and safe care [13]. 
Study showed that the patient should be monitored during food intake, and changes in respiratory rate, saturation and/or heart rate are signs of aspiration, whereas the return of the diet through the tracheostomy is a confirmation. When changes in these parameters arise, feeding should be discontinued and the patient monitored [14]. For the research subjects, patient acceptance was of higher priority than the monitoring of some of these signs.

Food reintroduction without defined criteria and uniform conduct by the team may lead the patient to clinical worsening, according to studies, by aspiration pneumonia, silent aspiration and bronchoaspiration, and these factors should be monitored during the process of food reintroduction by checking vital signs, performing laboratory tests and chest X-ray $[14,15]$. The sample had positive result, as most pointed pneumonia and bronchopneumonia as worsening of clinical conditions arising from food reintroduction without criteria. However, silent aspiration was reported by only $15 \%$, which shows that this clinical evidence is little known and/or monitored. As for the factors that should be monitored, the only factor barely mentioned by the sample was laboratory tests.

Most of the sample wrongly pointed the nutritionist as the professional who should prescribe the diet. It is known that the only professional who can perform medical prescription is the physician, whereas the nutritionist is responsible for dietary prescription. The dietary prescription is a responsibility of the nutritionist only and to elaborate this professional uses specific methods and therapeutic techniques, in which method is understood as a systematic set of procedures for the purpose of production and/or application of knowledge, and technique is the set of all the specific activities appropriate to the general principles outlined in the methodology [16].

The frequency with which this reintroduction process currently takes place for scholars is still quite small, which was evidenced by the subjects of this study, in which $60 \%$ of nurses reported that food reintroduction rarely occurs in units $[9,14,15]$.

\section{Conclusion}

The present research aimed to evaluate the nurses' knowledge on food reintroduction in tracheostomized patients, identifying their difficulties and risks of a food reintroduction with poorly defined criteria.

It is clear that this issue has been rarely addressed and that the shortage of literature and the limited approach on the subject during the academic training reflect on professional unpreparedness. This becomes evident by the lack of knowledge of nurses on the concomitant medical conditions that the patient must present so that the food reintroduction by mouth occurs.

However, a positive finding was that the indication by the multidisciplinary team was identified correctly by the vast majority of respondents, which reveals a change of structure within the units, where currently decisions are made after a consensus of the team, and not only by a single member.

We can also point out that, even without approach on the subject during training, most nurses have assertively pointed out what should be evaluated and the time in which the patient should be re-evaluated. The lack of knowledge about diet prescription was also found during the research. The only professional that can prescribe is the physician, whereas the dietary prescription is a responsibility of nutritionists, and this is not known by nurses.

The lack of criteria for the process of reintroducing food may cause several complications for the patient. Minimizing these complications is an essential role of the nurse; however, it has not been fully performed, as perceived by the research.

Considering that the analyzed results have showed that knowledge on the subject is scarce and the process has rarely happened in hos- 
pitals, this study recommends that this theme is approached since graduation and that continuing education measures are implemented in institutions providing care for the tracheostomized patient so that the reintroduction of oral diet occurs, wherever possible, safely.

\section{References}

1. Nettina SM. Prática de Enfermagem. Rio de Janeiro: Guanabara Koogan; 2011. 3(1): 209

2. Pinto DM, Schons ES, Busanello J, Costa VZ. Patient safety and the prevention of skin and mucosal lesions associated with airway invasive devices. Rev. esc. enferm. USP [Internet] 2015 [Cited 2016 Fev 27]:49(5). Available from: http://www.scielo.br/scielo. php? script=sci arttext\&pid=S0080-62342015000500775.

3. Sodhi K; Shrivastava A; Singla MK. Implications of dedicated tracheostomy care nurse program on outcomes. J Anesth [Internet] 2014 [Cited 2016 Fev 27]:28(3);374-80. Available from: http://pesquisa.bvsalud.org/portal/resource/pt/mdl-24097169.

4. Morris LL, Whitmer A, McIntosh E. Tracheostomy Care and Complications in the Intensive Care Unit. Crit Care Nurse [Internet] 2013 [Cited 2016 Fev 27]:33(5). Available from: http://pesquisa.bvsalud.org/portal/resource/pt/mdl-24085825.

5. Smeltzer SC; Bare BG. Tratado de Enfermagem Médico Cirúrgica. Rio de Janeiro: Guanabara Koogan; 2011. 646p.

6. Polit DF, Hungler BP. Fundamentos de pesquisa em enfermagem. Porto Alegre: Artes Médicas; 2011.

7. Lobiondo-Wood G, Haber J. Pesquisa em enfermagem: métodos, avaliação crítica e utilização. Rio de Janeiro: Ed. Guanabara Koogan; 2009, 5 ed.

8. Ministério da Educação. Diretrizes curriculares nacionais do curso de graduação em enfermagem. Available from: http://portal. mec.gov.br/cne/arquivos/pdf/Enf.pdf. Cited: 08 mar. 2016.

9. Joanne W. Assessing Dysphagia. Journal of Community Nursing. 2007; 21 (6).

10. Departamento de Nutrição e dietética. Protocolo de triagem do risco de disfagia. Universidade Estadual de Campinas UNICAMP. 2010.

11. Smith HCA, Goldstein LB. Cough and Aspiration of Food and Liquids Due to Oral-Pharyngeal. Dysphagia: ACCP EvidenceBased Clinical Practice Guidelines. American Speech Language Hearing Association - ASHA, 2006.

12. Guidelines for Swanllonwing and Nutrition. Dartford and Gravesham. Tracheostomy, 2003.
13. Dawson D. Essential principles: tracheostomy care in the adult patient. Nurs Crit Care [Internet] 2014 [Cited 2016 Fev 27]:19(2):63-72.Available from: http://pesquisa.bvsalud.org/ portal/resource/pt/mdl-24529106.

14. Clarence TS, Steven BL. Comments on Selected Recent Dysphagia Literature. Dysfhagia 2010; 25,345 -351.

15. Carlos VR, Brow MD, Kelli HM, Amy D, Mandaville MA, Paul E Channey MD, Guy SRN, Charlotte MD. Swallonwing dysfunction after mechanical ventilation in trauma patients. University Medical Center Brackenridge. Journal of Critical Care. 2011;26 (9).

16. Resolução CFN No 304, de 26-02-2003. Critérios Para Prescrição Dietética Na Área De Nutrição Clínica E Dá Outras Providências. Disponível em: http://www.cfn.org.br/novosite/ pdf/res/2000 2004/res304.pdf. Acesso em 08 de março de 2016.

\section{Publish in International Archives of Medicine}

International Archives of Medicine is an open access journal publishing articles encompassing all aspects of medical science and clinical practice. IAM is considered a megajournal with independent sections on all areas of medicine. IAM is a really international journal with authors and board members from all around the world. The journal is widely indexed and classified Q1 in category Medicine. 